\title{
Curcumin induces heme oxygenase-1 in normal human skin fibroblasts through redox signaling: Relevance for anti-aging intervention
}

\author{
Cristovao F. Lima ${ }^{1}$, Cristina Pereira-Wilson ${ }^{2}$ and Suresh I. S. Rattan ${ }^{3}$ \\ ${ }^{1} \mathrm{CITAB}$ - Centre for the Research and Technology of Agro-Environmental and Biological Sciences, Department of \\ Biology, University of Minho, Braga, Portugal \\ ${ }^{2}$ CBMA - Centre of Molecular and Environmental Biology, Department of Biology, University of Minho, Braga, \\ Portugal \\ ${ }^{3}$ Laboratory of Cellular Ageing, Department of Molecular Biology, Aarhus University, Aarhus - C, Denmark
}

Scope: Curcumin, a component of the spice turmeric, was tested for its potential hormetic anti-aging effects as an inducer of mild stress.

Methods and results: Early passage young human skin fibroblasts treated with low doses of curcumin (below $20 \mu \mathrm{M}$ ) showed a time- and concentration-dependent induction of heme oxygenase-1 (HO-1), followed by compensatory increase in glutathione-S-transferase activity, GSH levels and GSH/GSSG ratio. These effects were preceded by induction of oxidative stress (increased levels of reactive oxygen species and DNA damage) and impairment of cells' GSH redox state. Curcumin also induced nuclear factor-erythroid-2-related factor 2 accumulation in the nuclei. The use of the antioxidant $N$-acetyl cysteine prevented the induction of HO-1 by curcumin. Pharmacological inhibition of phosphatidylinositol 3-kinase, but not other kinases, significantly prevented curcumin-induced HO-1 levels, which was corroborated by the induction of phospho-Akt levels by curcumin. Late passage senescent cells already had higher HO-1 levels, and further induction of HO-1 by curcumin was considerably impaired. The induction of stress responses by curcumin in human cells led to protective hormetic effects to further oxidant challenge.

Conclusion: Curcumin induces cellular stress responses in normal human skin fibroblasts through phosphatidylinositol 3-kinase/Akt pathway and redox signaling, supporting the view that curcumin-induced hormetic stimulation of cellular antioxidant defenses can be a useful approach toward anti-aging intervention.

\section{Keywords:}

Anti-aging / Antioxidant defenses / Curcumin / Hormetic effects Normal human skin fibroblasts

\section{Introduction}

Several dietary factors have been linked to health and longevity. Fruits and vegetables are among the dietary components for which epidemiologic studies suggest an

Correspondence: Dr. Cristovao F. Lima, Department of Biology, University of Minho, Campus de Gualtar, 4710-057 Braga, Portugal

E-mail: lima@bio.uminho.pt

Fax: $+351-253678980$ association between their consumption and a decreased risk of premature death and mortality from certain age-related diseases [1]. Many of the phytochemicals present in plants

Abbreviations: ARE, antioxidant-response element; GST, glutathione-S-transferase; HIF-1, hypoxia inducible factor-1; HO-1, heme oxygenase-1; Hsp, heat shock protein; Keap1, Kelch-like ECH-associated protein 1; LDH, lactate dehydrogenase; MARS, maintenance and repair systems; NAC, $\mathrm{N}$-acetyl cysteine; Nrf2, nuclear factor-erythroid-2-related factor 2; PI3K, phosphatidylinositol 3-kinase; ROS, reactive oxygen species; tert-BOOH, tert-butyl hydroperoxyde 
are generally accepted as contributors toward these health positive effects. Besides other intrinsic properties of these compounds, such as being direct antioxidants, phytochemicals may also be beneficial by working as hormetins by mild stress-induced induction of pathways of protection, maintenance and repair $[2,3]$. Curcumin is one such phytochemical for which several studies already exist relating its health beneficial effects, mainly as an anti-inflammatory and anticancer compound at relatively high doses [4]. However, little is known about the effects and molecular mechanisms of action of curcumin on normal human cells undergoing aging.

Biological aging is a fundamental process where the progressive decline of organismal fitness and cellular functions represent major risk factors for the development of age-related diseases and eventual death. Substantial data support the view that the accumulation of intra- and extracellular molecular damage during the aging process results in part from the progressive failure of cellular maintenance and repair systems (MARS) [5]. Therefore, stimulating MARS has been increasingly recognized as an important approach for aging intervention and prevention [5, 6]. Mild stress-induced stimulation of MARS represents a promising strategy to achieve this goal. Such adaptive response of biological systems to a low or intermittent dose of otherwise harmful condition resulting in protection against subsequent stresses is known as hormesis [2, 7]. Some potential hormetic agents (hormetins), such as physical (radiation, temperature and exercise), chemical (hormones and prooxidants) and nutritional (food restriction and nutritional components) hormetins, have been tested and shown to bestow beneficial and anti-aging effects on various aging systems, including human cells $[3,8]$. We have recently reported that curcumin could be a hormetin since it was able to significantly increase heme oxygenease-1 (HO-1) expression, one cytoprotective enzyme under the influence of oxidative stress response, in normal human skin fibroblasts [9].

Curcumin (diferuloylmethane) is the active polyphenolic compound present in the rhizome of the Indian spice Curcuma longa Linn (turmeric, Zingiberaceae family), and it has been receiving increasing attention due to its ability to induce cellular stress responses as well as repair, detoxifying and antioxidant pathways. For example, curcumin was able to coinduce heat shock proteins (Hsps), in particular Hsp70, in rat cells when cotreated with various stresses, including heat shock [10]. Recently, curcumin alone was reported to induce the heat shock response in human leukemia cells, in particular causing the nuclear translocation of the heat shock transcription factor and increasing the expression of Hsp70 at transcriptional and translational levels [11]. From a biphasic dose-response hormetic view, curcumin at low concentrations was able to stimulate proteasome activity, but was inhibitory at high concentrations [12]. Several studies also report the ability of curcumin to induce protective proteins such as HO-1, phase II enzymes and glutamate cysteine ligase under the antioxidant-response element (ARE) [13-19]. Some of these studies showed that the induction of these enzymes conferred further protection against oxidative stress in endothelial [14], neuronal [13] and vascular smooth muscle [16] cells, corroborating curcumin's hormetic effects.

Several reports have shown that the induction of many of these detoxifying and antioxidant cytoprotective enzymes by curcumin is mediated by the nuclear factorerythroid-2-related factor 2 (Nrf2) through activation of the cis-acting element $\operatorname{ARE}[16,20]$. Also in vivo, oral administration of curcumin resulted in the induction of Nrf2-dependent genes in the liver and small intestine of rodents [21-23]. In unstressed cells, Nrf2 controls basal expression of its target genes and is constantly targeted by the Kelch-like ECH-associated protein 1 (Keap1) for degradation catalyzed by the 26S proteasome via the ubiquitindependent pathway [24]. Reactive chemical stresses or other ARE-inducers lead to Nrf2 accumulation in the nucleus that results in increasing transcription of many cytoprotective enzymes. Many studies showed that curcumin is able to stabilize Nrf2, leading to its accumulation in the nucleus. Inactivation of Keap1 through modifications of its cysteine thiols, and/or phosphorylation of serine or threonine residues of Nrf2 by different kinases has been suggested as possible molecular mechanisms behind Nrf2 stabilization by curcumin [25].

Therefore, since the induction of HO-1 by curcumin is indicative of its potential hormetic ability, we have undertaken studies to elucidate the effects of curcumin in normal human skin fibroblasts undergoing aging in vitro. Normal diploid human cells that undergo progressive aging during serial passaging in vitro and eventually become replicatively senescent have been used extensively to study the molecular basis of cellular aging [8]. In addition, this same model has been used to test the hormetic effects of different stressors at the level of various cellular functions [8].

\section{Materials and methods}

\subsection{Chemicals and antibodies}

Curcumin (1,7-bis[4-hydroxy-3-methoxyphenyl]-1,6-heptadiene-3,5-dione), DMEM, antibiotic/antimycotic solution, kinase inhibitors PD98059, SB203580, SP600125, LY294002, RO320432 and staurosporine, tert-butyl hydroperoxyde (tert-BOOH), anti- $\beta$-actin antibody as well as all other reagents were from Sigma-Aldrich (St. Louis, MO, USA), unless otherwise specified. Fetal bovine serum was bought from Lonza (Verviers, Belgium). 2',7'-Dichlorodihydrofluorescein diacetate was purchased from Molecular Probes (Eugene, OR, USA). Antibodies against HO-1, Hsp27, Hsp70 and Hsp90 were obtained from Stressgen (Victoria, British Columbia, Canada). Anti-Nrf2, anti-phospho-ERK1/2, anti-JNK, anti-phospho-JNK and anti-p38 
antibodies were purchased from Santa Cruz Biotechnology (Santa Cruz, CA, USA). Antibodies against phospho-Akt (Ser473), total Akt, p44/42 MAPK (ERK1/2) and phosphop38 MAPK (Thr180/Tyr182) were purchased from Cell Signaling (Danvers, MA, USA). Secondary antibodies HRP donkey anti-rabbit and sheep anti-mouse were purchased from GE Healthcare (Bucks, UK).

\subsection{Cell culture}

Normal diploid adult human skin fibroblasts (ASF-2 cells) isolated from a breast biopsy specimen of a consenting young healthy Danish woman (aged 28 years) [26] were used. ASF-2 cells were maintained at $37^{\circ} \mathrm{C}$ in a humidified $5 \%$ CO2 atmosphere grown in DMEM supplemented with $10 \%$ fetal bovine serum, $10 \mathrm{mM}$ Hepes and 1\% antibiotic/ antimycotic solution. Cell cultures were split in the ratio 1:2 or 1:4 after they became confluent. All experiments were performed with ASF-2 cells with cumulative populations doublings between 15 and 25 (25-45\% of replicative lifespan completed) at a density of 100000 cells/well in a 6-well plate, unless otherwise specified. Some experiments were also performed with late passage senescent ASF-2 cells with cumulative populations doublings around 45 ( $~ 90 \%$ of replicative lifespan completed) at a density of 50000 cells/ well in a 6-well plate. All experiments were performed $24 \mathrm{~h}$ after seeding using complete medium. Curcumin and kinase inhibitors were added to culture medium dissolved in DMSO (final concentration in the assays was $0.5 \% \mathrm{v} / \mathrm{v}$ ); controls received vehicle only.

\subsection{Cell viability and growth}

Necrosis induced by curcumin to human skin fibroblasts was evaluated visually under a phase contrast light microscope by severe morphological changes with appearance of blebs in the cytoplasmic membrane.

The effects of curcumin on cell viability and growth were evaluated by the MTT assay (Supporting Information).

In the experiment where the hormetic potential of curcumin was tested, ASF-2 cells were incubated for $2 \mathrm{~h}$ with $10 \mu \mathrm{M}$ or $20 \mu \mathrm{M}$ curcumin, and then the medium was removed and replaced with fresh medium. After $16 \mathrm{~h}$ of recovery, cells were incubated with $200 \mu \mathrm{M}$ tert-BOOH for $3 \mathrm{~h}$ and cell viability measured by the lactate dehydrogenase (LDH) leakage method as described previously [27].

\subsection{Reactive oxygen species levels}

The levels of reactive oxygen species (ROS) in ASF-2 cells treated with curcumin were measured using the $2^{\prime}, 7^{\prime}$-dichlorodihydrofluorescein diacetate probe following manufacturer's instructions (for details, see Supporting Information Materials and methods).

\subsection{Comet assay}

The potential of curcumin to induce DNA damage to ASF-2 cells was evaluated by the alkaline version of the comet assay as described previously [28]. Briefly, after treating cells for $1 \mathrm{~h}$ with different concentrations of curcumin $(100 \mu \mathrm{M}$ tert$\mathrm{BOOH}$ was used as a positive control), cells were resuspended using trypsin solution, embedded in $0.5 \% \mathrm{w} / \mathrm{v}$ low melting agarose and spread onto agarose-coated slides. Cells were then lysed to expose DNA, and the nucleoids electrophoresed for $20 \mathrm{~min}$ at $0.8 \mathrm{~V} / \mathrm{cm}, 300 \mathrm{~mA}$, in a cold room $\left(4^{\circ} \mathrm{C}\right)$. After neutralization, comet images were analyzed by visual scoring under a fluorescent microscope and 100 nucleoids per slide counted.

\subsection{GSH content}

Protein from cell scrapes was pelleted in 5\% w/v 5-sulfosalicyilic acid and the glutathione levels determined in the supernatant by the DTNB-GSSG reductase recycling assay were as described previously [29]. The results were expressed as nmol GSH/mg of protein.

\subsection{Glutathione-S-transferase activity}

For measurement of glutathione-S-transferase (GST) activity, cell homogenates were centrifuged at $10000 \times \mathrm{g}$ for $10 \mathrm{~min}$ at $4^{\circ} \mathrm{C}$ and the supernatant collected. The activity of GST was measured spectrophotometrically at $37^{\circ} \mathrm{C}$ as described previously [27], and the results were expressed as percentage from control.

\subsection{Protein extraction and Western blotting}

To determine the level of proteins of interest, ASF-2 cells were treated with different concentrations of curcumin for the indicated times, cell monolayers were washed twice with PBS and then lysed in ice-cold lysis buffer $(50 \mathrm{mM}$ Tris- $\mathrm{HCl}, \mathrm{pH} 8,150 \mathrm{mM} \mathrm{NaCl}, 0.5 \% \mathrm{v} / \mathrm{v} \mathrm{NP}-40,0.1 \%$ v/v SDS, $0.1 \% \mathrm{v} / \mathrm{v}$ deoxycholate, $1 \mathrm{mM}$ EDTA, $1 \mathrm{mM}$ EGTA) containing $1 \mathrm{mM}$ PMSF, $1 \times$ complete protease inhibitor cocktail (Roche, Mannheim, Germany) and phosphatase inhibitors $(20 \mathrm{mM}$ $\mathrm{NaF}, 20 \mathrm{mM} \mathrm{Na} \mathrm{V}_{3} \mathrm{O}_{4}$ ). When kinase inhibitors, $\mathrm{N}$-acetyl cysteine (NAC) and other antioxidants were used, they were pre-incubated for $30 \mathrm{~min}$ before addition of curcumin.

For the preparation of nuclear extracts, cells were washed twice with PBS and nuclear fractions prepared as described elsewhere [13] (for details, see Supporting Information). 
Protein concentration was quantified using the Bio-Rad DC protein assay (Bio-Rad Laboratories, Hercules, CA, USA) and BSA used as protein standard.

For Western blot, $20 \mu \mathrm{g}$ of protein was resolved in SDSpolyacrylamide gel and then electroblotted to a Hybond-P polyvinylidene difluoride membrane (GE Healthcare). Membranes were blocked in TPBS (PBS with $0.05 \%$ Tween20) containing $5 \% \mathrm{w} / \mathrm{v}$ nonfat dry milk, washed in TPBS and then incubated with primary antibody. After washing, membranes were incubated with secondary antibody conjugated with IgG horseradish peroxidase and immunoreactive bands were detected using the Immobilon solutions (Millipore, Billerica, MA, USA) under a chemiluminescence detection system, the Chemi Doc XRS (Bio-Rad Laboratories). Band area intensity was quantified using the Quantity One software from Bio-Rad. $\beta$-Actin was used as loading control.

\subsection{Statistical analysis}

Data were presented as mean \pm SEM. Statistical differences among data groups were analyzed by one-way ANOVA followed by the Student-Newman-Keuls multiple comparison test, or analyzed by the Student's $t$-test when appropriate, using GraphPad Prism 4.0 software (San Diego, CA, USA). Differences between groups were considered significant when $p$-values were $\leq 0.05$.

\section{Results}

\subsection{Curcumin affects cell viability and growth}

Exposure of normal adult human skin fibroblasts ASF-2 to 5-20 $\mu \mathrm{M}$ curcumin for up to 3 days inhibited cell proliferation in a concentration-dependent manner, as determined by the cell's capacity to reduce MTT (Supporting Information Fig. 1A), and by counting the cell numbers (Supporting Information Fig. 1B). The extent of cell growth inhibition by curcumin was statistically significant only at $10 \mu \mathrm{M}(36 \%$ in the MTT assay and 65\% in cell counting) and above ( $87 \%$ inhibition at $20 \mu \mathrm{M})$. Treatment with $30 \mu \mathrm{M}$ curcumin for $24 \mathrm{~h}$ resulted in significant cell death by necrosis as evident from severe morphological changes and the appearance of blebs in the cytoplasmic membranes (data not shown).

\subsection{Curcumin induces HO-1 levels in human skin fibroblasts}

Previous studies have shown the ability of curcumin to induce stress-associated proteins, such as HO-1 and Hsps, in different cell types, or to work as a coinducer of Hsps (Section 1). Therefore, the ability of curcumin to induce the levels of HO-1 (also known as Hsp32) and Hsps in normal diploid human skin fibroblasts was studied by Western blot. Treatment of ASF-2 cells with $10 \mu \mathrm{M}$ curcumin for $24 \mathrm{~h}$ remarkably induced the levels of HO-1 in ASF-2 cells, but did not change significantly Hsp27, Hsp70 and Hsp90 levels (Supporting Information Fig. 2A). Curcumin was also unable to coinduce the levels of these Hsps after a heat shock treatment of $1 \mathrm{~h}$ at $41^{\circ} \mathrm{C}$ (Supporting Information Fig. 2A). Induction HO-1 levels in ASF-2 cells by curcumin were in a concentration- and time-dependent manner (Figs. 1A and $\mathrm{B})$.

\subsection{Curcumin increases ROS and induces DNA damage}

In view of the induction of HO-1 stress protein, we next asked whether this would be the result of oxidative stress imposed on the cells by curcumin in the first minutes of incubation before it becomes greatly metabolized. Incubation of ASF-2 cells with curcumin for 30 min resulted in a

A Effect of concentration (24 h)

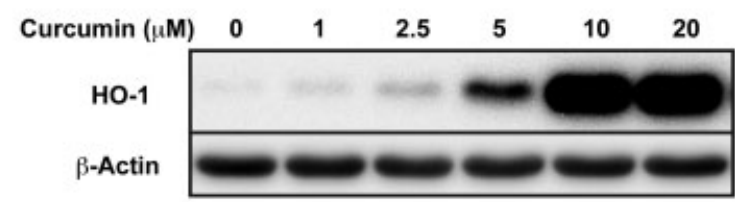

B Effect of incubation time

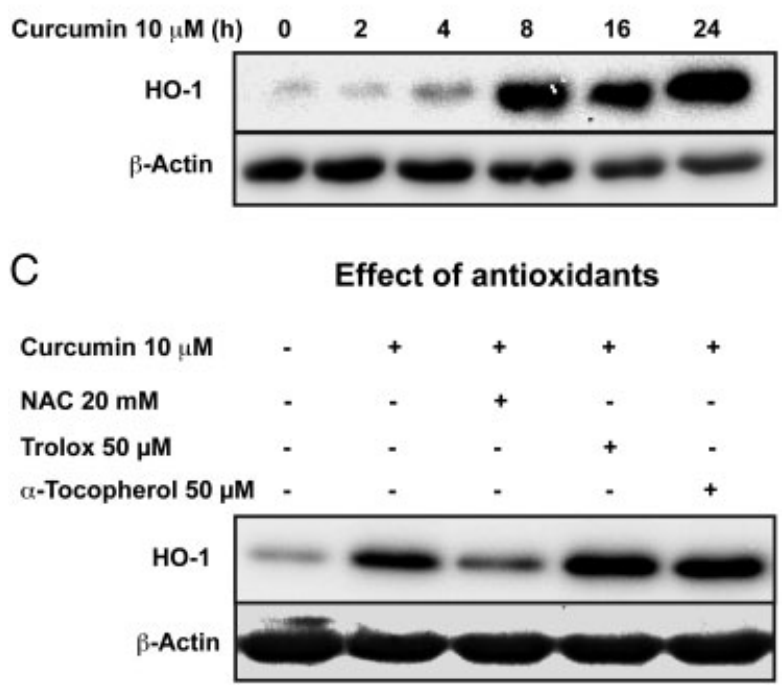

Figure 1. Effect of curcumin on the levels of HO-1 in ASF-2 cells, at different concentrations for $24 \mathrm{~h}(\mathrm{~A})$, or with $10 \mu \mathrm{M}$ curcumin for different incubation times (B). (C) Cells were incubated with $10 \mu \mathrm{M}$ curcumin with or without the antioxidants NAC, trolox or $\alpha$-tocopherol for $8 \mathrm{~h}$, and the levels of HO-1 measured by Western blot. Blots are representative of three (A and $B$ ) or two (C) independent experiments. $\beta$-Actin was used as loading control. 
A ROS levels (30 $\mathrm{min})$

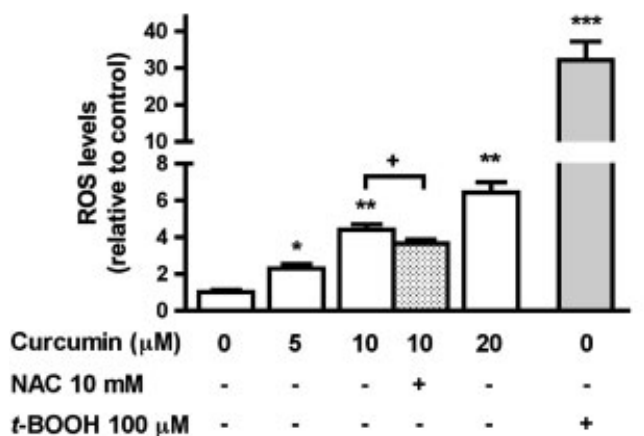

B

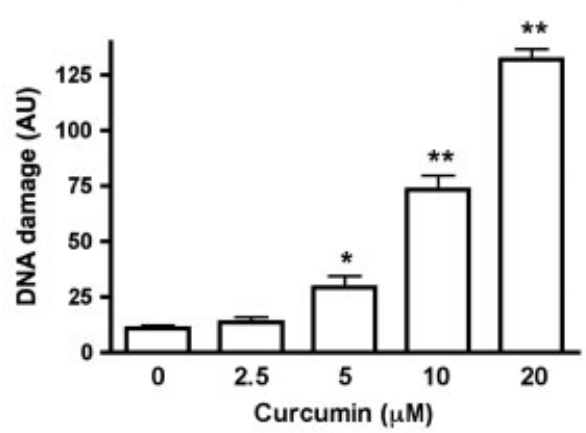

C

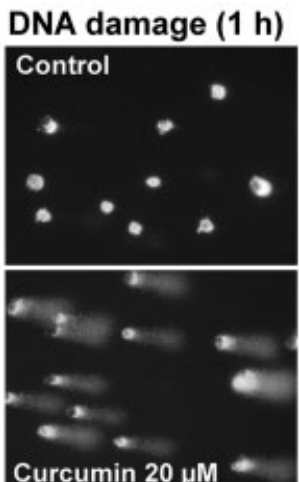

Figure 2. Curcumin increases ROS levels and DNA damage in ASF-2 cells. (A) Effect of different concentrations of curcumin for 30 min in ROS levels as measured by fluorometry using the DCF probe; $t-\mathrm{BOOH} 100 \mu \mathrm{M}$ was used as a positive control. (B) Effect of different concentrations of curcumin for $1 \mathrm{~h}$ on DNA damage measured by the alkaline version of the comet assay. (C) Representative images of the comet assay in the control and $20 \mu \mathrm{M}$ curcumin conditions. ( $\mathrm{A}$ and $\mathrm{B}$ ) Values are mean $\pm \mathrm{SEM}$ of at least three independent experiments. ${ }^{*} p \leq 0.05 ;{ }^{* *} p \leq 0.01$ when compared with control by the one-way ANOVA. ${ }^{* * *} p \leq 0.001$ when compared with control by the Student's $t$-test. ${ }^{+} p \leq 0.05$ when compared with each other by the Student's $t$-test.

concentration-dependent significant increase in ROS levels, as measured by the DCF probe (Fig. 2A). In addition, using the alkaline version of the comet assay, curcumin showed induction of DNA damage in a concentration-dependent manner after $1 \mathrm{~h}$ of incubation (Fig. 2B). When incubated with $20 \mu \mathrm{M}$ curcumin, almost $100 \%$ cells showed mild or moderate DNA damage (Fig. 2C). Therefore, in the short term curcumin seems to induce mild oxidative stress to the cells that did not result in cell death, but which may be the trigger for the induction of a stress response (as shown by a later induction of HO-1 levels) accompanied by the stop of cell cycle. On the other hand, the oxidant $t$-BOOH (used as positive control) induced much higher levels of ROS (Fig. 2A) and DNA damage (data not shown), which then results in extensive cell death (Fig. 5C).

\subsection{NAC prevents curcumin-induced HO-1 levels}

To explore whether the increase of ROS was involved in the expression of HO-1 induced by curcumin, coincubations with well-known antioxidants were performed. As shown in Fig. 1C and Supporting Information Fig. 2B, the antioxidant NAC remarkably prevented the curcumin-induced increase of HO-1 (by about 60-80\%), as shown both by Western blot and by immunofluorescence. However, other antioxidants of reference with antiradical activity, such as trolox (a watersoluble vitamin E derivative) and $\alpha$-tocopherol (an absorbable vitamin $\mathrm{E}$ form), were unable to inhibit the induction of HO-1 by curcumin. In addition, ROS levels induced by $10 \mu \mathrm{M}$ curcumin were only slightly decreased (about 17\%) when cells were coincubated with NAC (Fig. 2A). As NAC, besides being a direct free radical-scavenger compound, as an analogue of cysteine is also a precursor for GSH synthesis and, therefore, able to increase cells' thiol-disulfide redox state, the overall results with the antioxidants indicate that the induction of HO-1 by curcumin may be due to the induction of cysteine-related redox stress rather than induction of ROS levels. In addition, since NAC was used in a high concentration, it may directly inactivate curcumin's reactivity against cellular protein and nonprotein cystein thiol groups.

\subsection{Redox signaling is modulated by curcumin}

To determine if curcumin affects the thiol-disulfide redox state of ASF-2 cells, the levels of the redox couple GSH and GSSG were measured. As shown in Fig. 3A, after $1 \mathrm{~h}$ of incubation with $10 \mu \mathrm{M}$ curcumin, a significant $10 \%$ decrease in GSH levels was observed, and continued to decrease until $4 \mathrm{~h}$ of incubation. However, after 16 and $24 \mathrm{~h}$ of curcumin incubation, GSH levels increased to more than double (Fig. 3A). GSSG accompanied this increase (Fig. 3B) but to a lower extent, which resulted after $24 \mathrm{~h}$ of incubation with a more than $50 \%$ increase in the GSH/GSSG ratio (Fig. 3C). Due to the decrease of GSH levels in the first hours of curcumin incubation, GSH/GSSG ratio also progressively decreased until $4 \mathrm{~h}$ before the significant increase by the end of incubation period. This indicates that curcumin affects negatively the cells' sulfhydryl groups at first (by inducing their oxidation or by its direct binding), and then cells respond by increasing glutathione synthesis to restore and increase the cells' thiol-disulfide redox state, a typical biphasic response observed in adaptive stress responses. The impairment of thiol-disulfide redox state in the first hours of curcumin incubation may influence redox signaling. In fact, the enzyme glutamate cysteine ligase involved in GSH synthesis, HO-1 and GST (a phase II enzyme, whose activity was also significantly increased by curcumin 


\section{A}

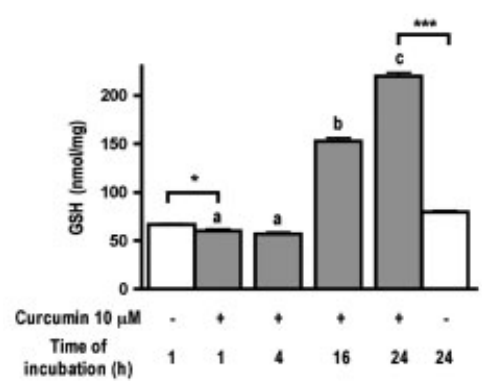

B

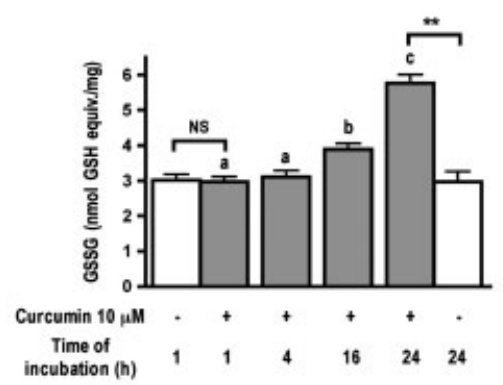

C GSH/GSSG ratio

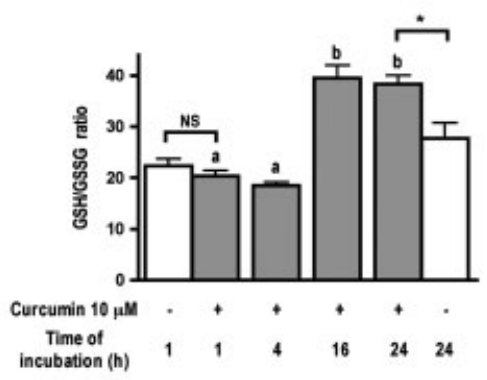

D

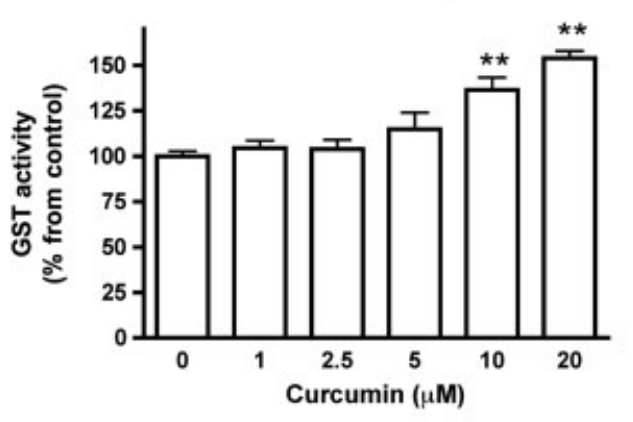

E

Nrf-2 expression

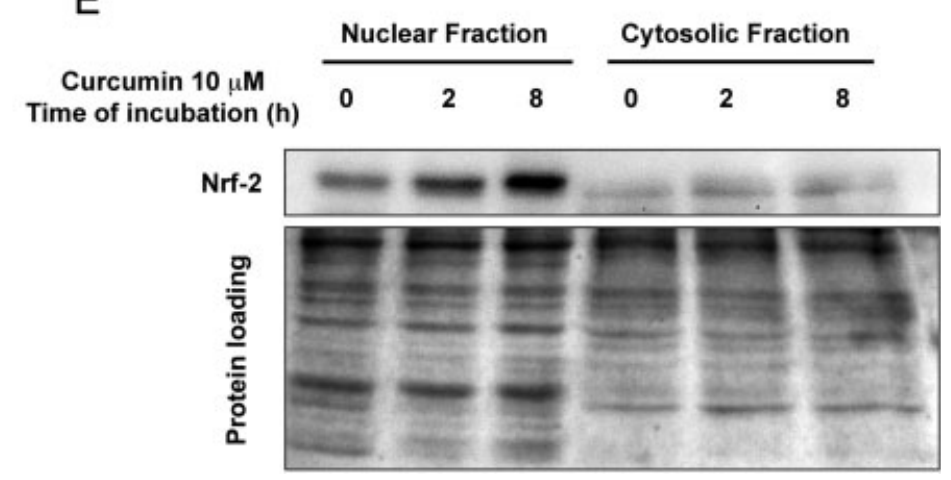

Figure 3. Effects of curcumin on ASF-2 cells' GSH content and redox signaling. (A, B and C) Cells were incubated with $10 \mu \mathrm{M}$ curcumin for different times and GSH and GSSG levels measured. Values are mean \pm SEM of at least three independent experiments. ${ }^{*} p \leq 0.05$, ${ }^{* *} p \leq 0.01$ and ${ }^{* * *} p \leq 0.001$ when compared with each other by the Student's $t$-test. NS, not significant $(p>0.05)$ when compared with each other by the Student's $t$-test. Letter notations: Groups with the same letter notation within the curcumin-treated samples are not significantly different from each other $(p>0.05)$ when analyzed by the one-way ANOVA. (D) Cells were incubated with curcumin at different concentrations for $24 \mathrm{~h}$ and the activity of GST measured. Values are mean \pm SEM of at least 3 independent experiments. ${ }^{* *} p \leq 0.01$ when compared with control by the one-way ANOVA. (E) Cells in $60 \mathrm{~mm}$-dishes $(4.5 \mathrm{~mL})$ were incubated with $10 \mu \mathrm{M}$ curcumin for different times, nuclear extracts isolated and Nrf2 expression measured by Western blot. Blot is representative of two independent experiments.

treatment in a concentration-dependent manner - Fig. 3D) are all part of a set of protective enzymes that are under the influence of a redox-sensitive transcription factor - Nrf2 $[25,30]$. The nuclear levels of Nrf2 were measured by Western blot and, as shown in Fig. 3E, curcumin induced a remarkable increase of this transcription factor in the nuclear fraction after 2 and $8 \mathrm{~h}$ of incubation. Therefore, the induction of cysteine-related redox stress by curcumin in the first hours of incubation seems to be involved in the increase of HO-1 expression and GST activity as well as in the increase of GSH content.

\subsection{Phosphatidylinositol 3-kinase/Akt pathway is involved in curcumin-induced HO-1 expression}

Many kinases such as phosphatidylinositol 3-kinase (PI3K), PKC, JNK, ERK and p38 may interfere with the phosphorylation of Nrf2 that is assumed to facilitate its accumulation in the nucleus to stimulate gene expression [25, 31]. ASF-2 cells were, therefore, incubated with curcumin in the presence of different kinase inhibitors for $8 \mathrm{~h}$ and the expression of HO-1 measured by Western blot. As shown in Fig. 4A, only the PI3K inhibitor LY decreased significantly the expression of HO-1 induced by curcumin. The inhibitors of MAPKK (in MAPK/ERK pathway), JNK and p38 MAPK (PD, SP and SB, respectively) did not modify the effect of curcumin on HO-1 levels. The same happened with RO, an inhibitor of PKC. Staurosporine was also used to inhibit PKC, and a decrease in HO-1 expression was observed (Fig. 4A), but associated with cell death. To confirm the involvement of PI3K/Akt pathway in the induction of HO-1 by curcumin, the expression of phosphorylated (active) form of Akt was measured. Contrary to what had happened with phospho (p)-ERK, p-JNK and p-p38 where no effect was observed, cells incubated with curcumin (for $8 \mathrm{~h}$ ) increased the expression of p-Akt (Fig. 4B) corroborating the previous results.

To explore if there was some dependence on the duration of curcumin exposure on p-Akt levels, incubations in the 
A

Involvement of kinases

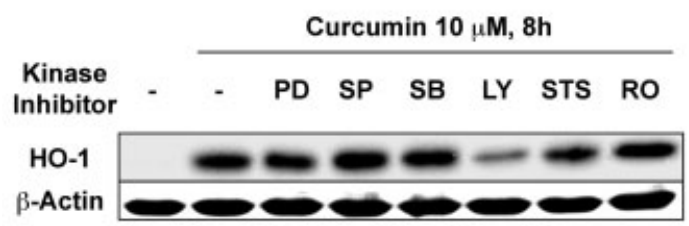

C

\section{Effect of time and inhibitors}

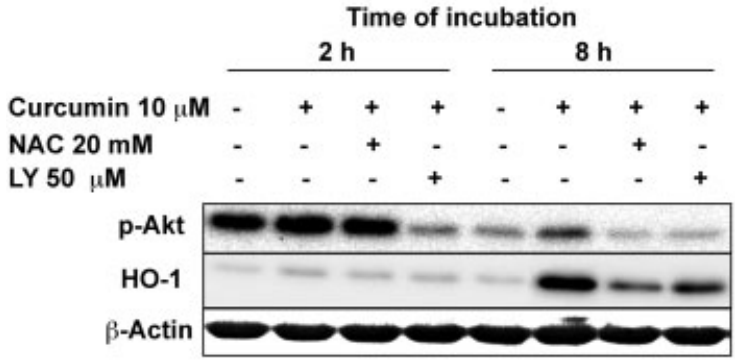

B

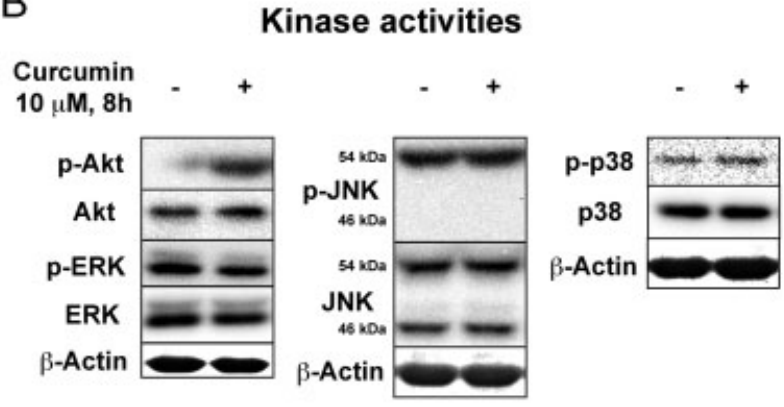

E

Effect of medium change

\section{Effect of time on p-Akt expression}
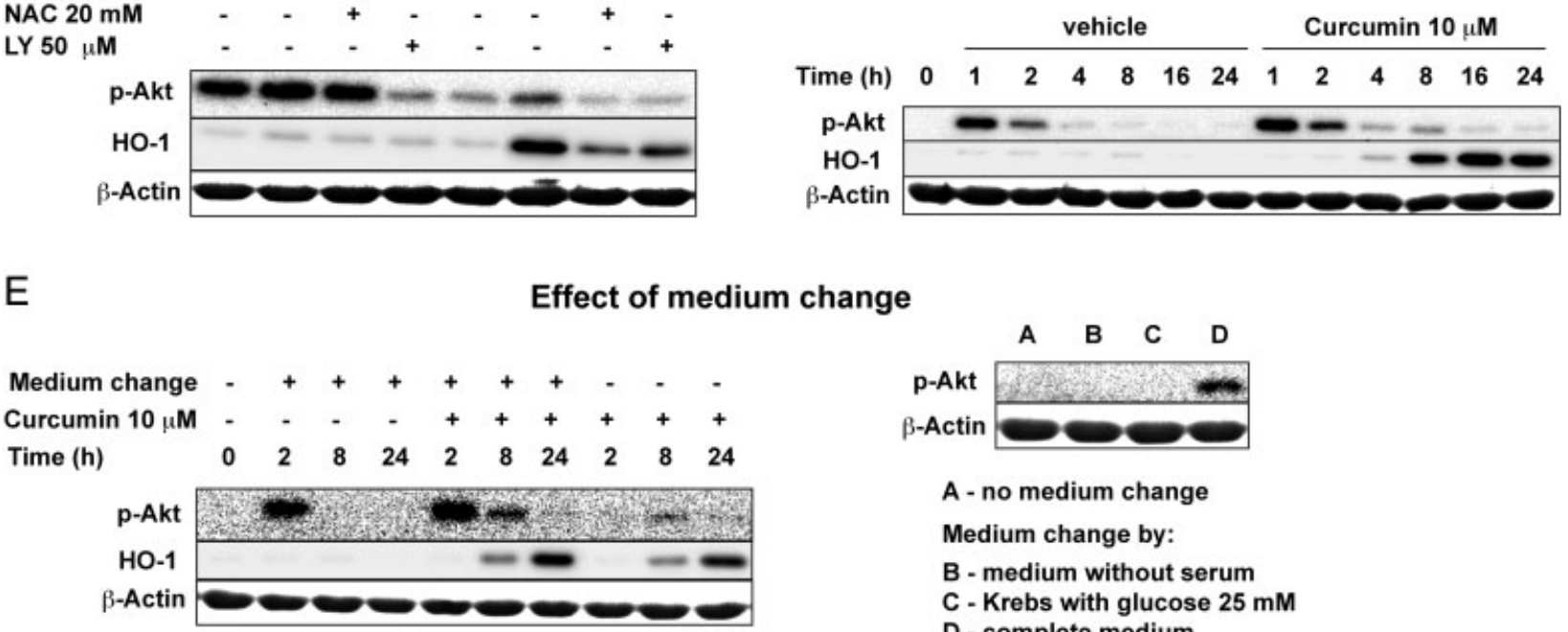

A - no medium change

Medium change by:

B - medium without serum

C - Krebs with glucose $25 \mathrm{mM}$

D - complete medium

Figure 4. Involvement of kinases in curcumin-induced HO-1 expression in ASF-2 cells. Cells were incubated with $10 \mu \mathrm{M}$ curcumin in the presence of different inhibitors, and after the time specified in the figures protein was extracted for Western blot. Inhibitors were added $30 \mathrm{~min}$ before curcumin. Blots are representative of at least two independent experiments. $\beta$-Actin was used as loading control. (A) Effect of different kinase inhibitors on curcumin-induced HO-1 expression: PD, PD98059 $50 \mu \mathrm{M}$; SP, SP600125 $10 \mu \mathrm{M}$; SB, SB203580 $10 \mu \mathrm{M}$; LY, LY294002 $50 \mu \mathrm{M}$, STS, staurosporine $50 \mathrm{nM}$; and RO, RO320432 $1.25 \mu \mathrm{M}$. Inhibitors alone did not induce HO-1 expression (data not shown). (B) Effect of curcumin for $8 \mathrm{~h}$ on the active (phosphorylated) form of different kinases. (C) Effect of curcumin in the presence of NAC or LY in the expression of phospho (p)-Akt after 2 and $8 \mathrm{~h}$ of incubation. (D) Phospho-Akt expression at indicated times after medium change in the absence or presence of $10 \mu \mathrm{M}$ curcumin. (E) Left panel: Effect of medium change and curcumin on the levels of phospho-Akt and HO-1; right panel: Effect of different components of the medium on p-Akt levels $2 \mathrm{~h}$ after replacing the medium.

presence of either LY or NAC for 2 and $8 \mathrm{~h}$ were performed. As shown in Fig. 4C, the expression of p-Akt was remarkably higher after $2 \mathrm{~h}$ of incubation than after $8 \mathrm{~h}$ of incubation, even in the absence of curcumin. As expected, LY decreased significantly p-Akt levels. NAC did not affect p-Akt levels after $2 \mathrm{~h}$ of curcumin incubation, but surprisingly decreased it after $8 \mathrm{~h}$ (Fig. 4C). Considering the effect of time on p-Akt levels, its expression along the time was determined in the absence and presence of curcumin. As shown in Fig. 4D, when medium was replaced by fresh medium containing curcumin or vehicle, p-Akt levels increased remarkably in the first hour and then began to decrease continuously in the following hours. The p-Akt level attained after $1 \mathrm{~h}$ was higher in the presence of curcumin, and the subsequent decrease on p-Akt levels was slowed down when curcumin was present (Fig. 4D).

Since an inhibition of PI3K/Akt pathway decreases HO-1 induction by curcumin and changing cells' medium induces this pathway (observed by the increase of p-Akt levels), we next asked if the effects of curcumin on HO-1 expression were dependent on medium change. For that, curcumin was added to the cells in the same medium they were in previously, and the expression of HO-1 and p-Akt was measured. As shown in Fig. 4E (left panel), curcumin added to cells without medium change also induced HO-1, although to a smaller extent than with medium change. This happened without an increase in $\mathrm{p}$ Akt at $1 \mathrm{~h}$ (data not shown) and $2 \mathrm{~h}$ of curcumin incubation 
(Fig. 4E); only at $8 \mathrm{~h}$ of incubation, a slight increase of p-Akt was observed. To determine the medium component that is responsible for the initial increase of p-Akt after medium change, medium of ASF-2 cells was replaced by fresh complete culture medium, by culture medium without serum or by Krebs buffer containing $25 \mathrm{mM}$ glucose. As shown in Fig. 4E (right panel), glucose and other components of the culture medium were not involved in the activation of PI3K/Akt pathway in ASF-2 cells, which happened only in the presence of serum.

\subsection{Curcumin increases stress tolerance in ASF-2 cells}

Considering the stress response elicited by curcumin in ASF2 cells, we next tested whether this effect would condition cells for a following oxidant challenge. For that, cells were incubated with curcumin for $2 \mathrm{~h}$, followed by a recovery period of $16 \mathrm{~h}$ where medium was removed and replaced by fresh one, and then exposed to the oxidant tert-BOOH. This pulse incubation with curcumin induced significantly HO-1 expression with maximal levels attained $4 \mathrm{~h}$ after the beginning of the recovery period, but decreasing after $16 \mathrm{~h}$ (Fig. 5A). The pulse treatment also induced significantly GSH levels after the $16 \mathrm{~h}$ recovery period, without significant changes in GSH/GSSG ratio and GSSG levels (Fig. 5B). As shown in Fig. 5C, the stress response induced by curcumin incubation for $2 \mathrm{~h}$ afforded $16 \mathrm{~h}$ later a significant protection against tert-BOOH-induced cell death, dependent on curcumin concentration, demonstrating its hormetic effects via a further oxidant challenge.

\subsection{Effects of curcumin on senescent ASF-2 cells}

To test if curcumin also induces HO-1 expression in late passage senescent cells, ASF-2 cells with $\sim 90 \%$ replicative lifespan completed were used. As shown in Fig. 6, senescent cells had already high expression levels of HO-1 in the control condition. Curcumin induced HO-1 expression in a concentration- and time-dependent manner in senescent cells (Figs. 6B and $\mathrm{C}$, respectively) but to a smaller extent than in young cells (Fig. 6A), which possess a higher proliferating rate. Medium change at the time of curcumin addition to the cells also induced the expression of p-Akt. Curcumin extended the high levels of p-Akt for a longer time (until $8 \mathrm{~h}$; Fig. 6C), also to a higher extent than that observed in young cells. As in young cells, NAC and LY inhibited the curcumininduced HO-1 expression (Fig. 6D). LY inhibited p-Akt levels from the beginning of the incubation, and NAC did the same but only after $8 \mathrm{~h}$ of incubation. Interestingly, LY in the presence of curcumin decreased the HO-1 expression levels below the control values after $24 \mathrm{~h}$ of incubation (Fig. 6D).
A

HO-1 expression

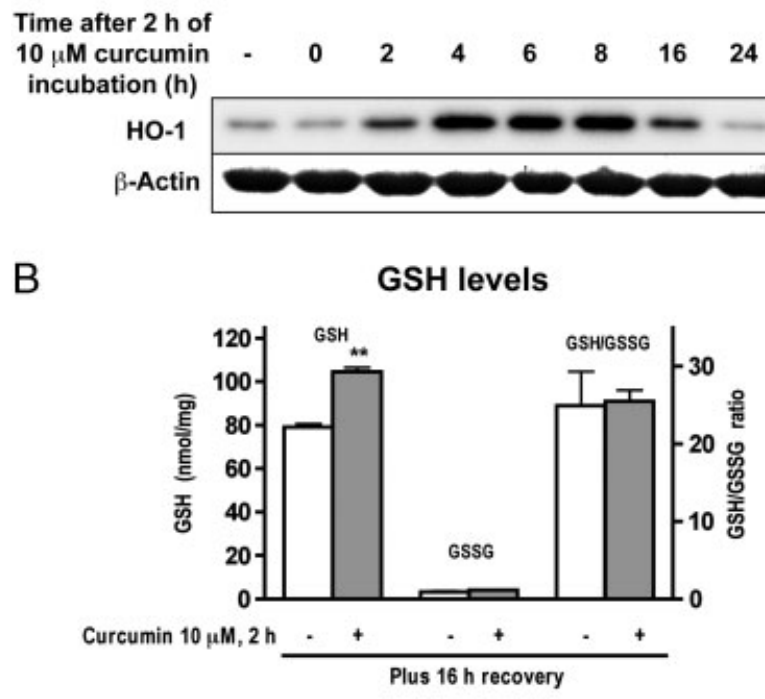

C

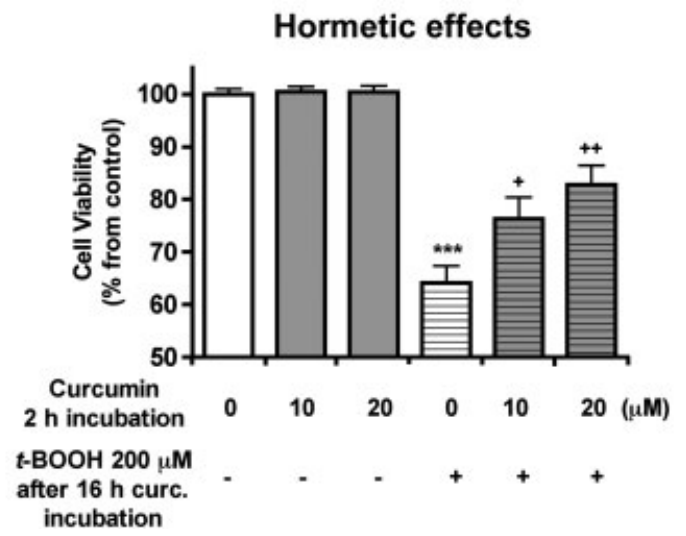

Figure 5. Hormetic effects of curcumin in ASF-2 cells. (A) Cells were incubated with $10 \mu \mathrm{M}$ curcumin for $2 \mathrm{~h}$, followed by a recovery period for different times with fresh medium, and the expression of HO-1 was measured by Western blot. Blots are representative of two independent experiments. $\beta$-Actin was used as loading control. (B) Cells were incubated with $10 \mu \mathrm{M}$ curcumin for $2 h$, followed by a recovery period of $16 h$ with fresh medium, and GSH and GSSG levels were measured as described in Section 2. Values are mean \pm SEM of three independent experiments. ${ }^{* *} p \leq 0.01$ when compared with the respective control by the Student's $t$-test. (C) Cells were incubated with 10 or $20 \mu \mathrm{M}$ curcumin for $2 \mathrm{~h}$, followed by a recovery period of $16 \mathrm{~h}$ with fresh medium. Then, cells were incubated with $200 \mu \mathrm{M}$ $t$-BOOH for $3 \mathrm{~h}$ and cell viability was measured by the LDH leakage method. Values are mean \pm SEM of at least four independent experiments. ${ }^{* * *} p \leq 0.001$ when compared with control by the Student's $t$-test. ${ }^{+} p \leq 0.05,{ }^{++} p \leq 0.01$ when compared with $t-\mathrm{BOOH}$ alone by the one-way ANOVA.

\section{Discussion}

Phytochemicals, such as phenolic compounds, are considered to contribute to the positive health benefits of fruits, vegetables and spice consumption [1, 32], and are proposed 
A

Young vs Senescent cells

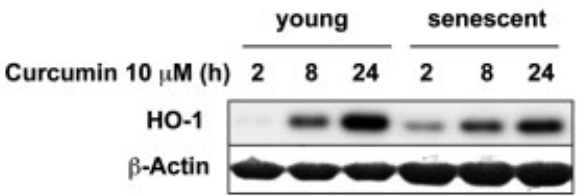

B Effect of concentration ( $24 \mathrm{~h})$

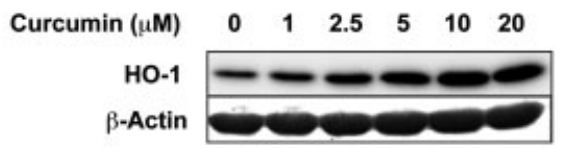

\section{Effect of incubation time}

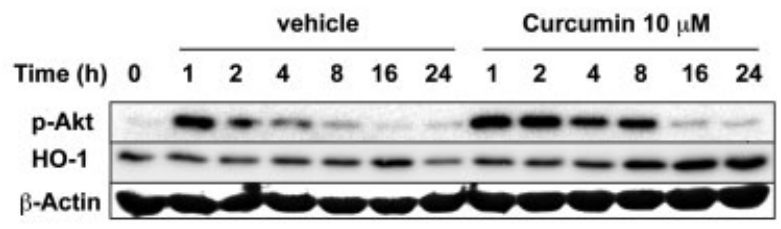

\section{Effect of time and inhibitors}

\begin{tabular}{|c|c|c|c|c|c|c|c|c|c|c|c|c|c|}
\hline Time (h) & 0 & & 2 & & & & 8 & & & & 2 & & \\
\hline Curc $10 \mu \mathrm{M}$ & - & - & + & + & + & - & + & + & + & - & + & + & + \\
\hline LY $50 \mu \mathrm{M}$ & - & - & - & + & - & - & - & + & - & - & - & + & - \\
\hline NAC $20 \mathrm{mM}$ & - & - & - & - & + & - & - & - & + & - & - & - & + \\
\hline p-Akt & & 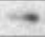 & - & & - & & - & & & 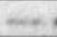 & - & & \\
\hline $\mathrm{HO}-1$ & - & - & 10 & - & - & - & - & - & - & - & & & - \\
\hline
\end{tabular}

Figure 6. Effects of curcumin on the levels of HO-1 in senescent ASF-2 cells. (A) Effect of curcumin on HO-1 levels in young and senescent cells. Senescent cells were incubated with curcumin at different concentrations for $24 \mathrm{~h}$ (B), or with $10 \mu \mathrm{M}$ curcumin for different incubation times (C), and the expression of HO-1 and p-Akt was measured by Western blot. (D) Effects of curcumin in the presence of NAC or LY on the levels of p-Akt and HO-1 after 2,8 and $24 \mathrm{~h}$ of incubation. Blots are representative of two independent experiments. $\beta$-Actin was used as loading control.

to be a good source of hormetic compounds, termed hormetins [33]. Here, we have tested the effects of curcumin in normal diploid human skin fibroblasts undergoing aging in vitro. We showed that curcumin worked as an hormetin by inducing mild oxidative stress as shown by increased levels of ROS, DNA damage and decrease of GSH content followed by enhanced antioxidant defenses, such as HO-1 levels, GST activity, GSH levels and GSH/GSSG ratio. The use of the antioxidant NAC prevented the induction of HO-1 by curcumin. This, together with the decrease of GSH content and increased accumulation of Nrf2 in nuclei in the first hours, suggest that a cellular thiol-disulfide redox state disturbance may cause the induction of a stress response by curcumin, rather than a sole increase of ROS levels. The stress response elicited by curcumin in human skin fibro- blasts led to hormetic effects via a further oxidant challenge with tert-BOOH, which can be beneficial in the process of aging where the cell's own pathways of protection, removal, maintenance and repair are impaired. In fact, an everincreasing body of evidence shows that low doses of stressors (mild stress) at young age can increase longevity and delay aging or protect from severe stress at old age [3, 34]. As individuals age, there is a gradual decline of cellular antioxidant defenses and a reduced ability of these systems to be induced by different stimuli [35]. The decreased ability of senescent fibroblasts to induce HO-1 levels upon stimulation with curcumin is a sign that aged and senescent cells are also impaired in their pathways of cellular stress responses, specially with respect to HO-1 induction, which is in accordance with the reports of studies in rodents where aging affected its stimulation by different stimuli [36-39].

The antioxidant, phase II and protective enzymes that are induced by curcumin treatment have been shown to be under the influence of the cis-acting element ARE, which are regulated by the redox-sensitive transcription factor Nrf2 $[24,25]$. Several studies both in vivo and in vitro have shown that, in fact, curcumin activates Nrf2 signaling [16, 20-23]. In human skin fibroblasts, we also observed an accumulation of Nrf2 in the nuclear fraction, suggesting that the induction of HO-1 and GST by curcumin was also through Nrf2 signaling. The increase of GSH levels and GSH/GSSG ratio observed after $24 \mathrm{~h}$ incubation was also attained probably through curcumin-induced increase in Keap1/Nrf2/ ARE axis signaling, since the rate-limiting enzyme in glutathione synthesis glutamate cysteine ligase (formerly known as $\gamma$-glutamylcysteine synthetase) is also under the influence of this redox-sensitive signaling pathway [30, 40]. It is known that Keap1 controls Nrf2 stability, which under nonstressing conditions constantly targets Nrf2 for degradation, representing an attractive target for controlling Nrf2 signaling [24]. Keap1 contains cysteine residues that are believed to regulate its association with Nrf2. Since curcumin is chemically reactive and able to act as Michael reaction acceptor, its ability to increase the cellular levels of ROS or its direct potential to oxidize cysteine groups on Keap1, and thus inhibiting it, is argued as the possible mechanisms that lead to Nrf2 stabilization [17, 25, 41, 42]. Based on our results, the latter hypothesis seems more likely.

A decrease in the levels and transcriptional activity of Nrf2 with age has been reported by different authors [43-45] and may explain the decrease in the levels of several antioxidant and protective enzymes and the age-related loss of GSH synthesis [43]. Experiments in human skin fibroblasts [46], and measurements in different organs of rats and mice [47-49] showed that cellular GSH/GSSG ratio declines during the aging process, due to a decrease in GSH and/or an increase in GSSG levels. Other studies also showed a decrease of antioxidant enzymes in mice astrocytes [44] and rat livers [45]. Therefore, the ability of curcumin to induce antioxidant and protective enzymes as well as GSH levels through Nrf2 signaling, besides protecting against oxidative 
stressors, may also delay the aging process or prevent the appearance of age-related abnormalities or diseases. In agreement with this, feeding mice with tetrahydrocurcumin (one of the major metabolites of curcumin) significantly increased their average and maximal lifespan [50]. In addition, rats fed with curcumin also present several anti-aging effects in neural tissue with increased activity of antioxidant enzymes and decreased content of lipid peroxides and lipofuscin [51]. Therefore, it would be interesting to further test the potential of regular hormetic exposure of normal human skin fibroblasts to curcumin in order to increase cellular stress response in old cells and to afford anti-aging properties. However, care should be taken in that approach, mainly with respect to the dose and time of exposure with curcumin, since aberrant induction of oxidative stress and Akt signaling may induce premature senescence [52, 53]. Nevertheless, increased Nrf2 signaling in Drosophila has shown to increase significantly their oxidative stress tolerance, as well as their lifespan [54]. Thus, if induction of Nrf2 signaling can be achieved by curcumin without significant changes on Akt signaling and ROS levels, anti-aging properties may be attainted by curcumin treatment.

Basal HO-1 expression levels in senescent fibroblasts were, however, much higher than in young cells. This is in accordance with in vivo data in liver and brain of rodents $[36-38,55]$ as well as in brain of humans [56], where HO-1 expression increases with age. That may be explained because HO-1 gene is under the influence of the activity of other transcription factors that may respond differently to aging as compared with Nrf2. Actually, it was previously reported that the DNA binding activity of the hypoxia inducible factor-1 (HIF-1) increases during aging along with the increase of the expression of HIF-1-dependent genes, including HO-1 [36]. Interestingly, inhibiting PI3K with LY for $24 \mathrm{~h}$ in senescent cells decreased HO-1 expression below the basal levels found in control condition. This agrees with different studies that report that PI3K/Akt pathway is involved in HIF-1 signaling [57, 58].

Another mechanism reported to be involved in Nrf2 stabilization is the phosphorylation of serine or threonine residues of the transcription factor by different kinases, such as PI3K, stress-induced MAPK and PKC [24, 25]. Different studies reported that curcumin stabilizes Nrf2 and/or induced HO-1 or other protective enzymes through some of these kinases [15-17, 20, 59]. The implications of kinase(s) among those studies are, however, not consistent, probably because cancer cell lines and tissues/cells from different mammalian species were used. Therefore, the use of normal human skin fibroblasts in this study will facilitate the association of our results with human aging and the prevention of age-related diseases. In ASF-2 cells, induction of HO-1 seems to be through PI3K/Akt pathway, since p-Akt levels are increased by curcumin and inhibiting PI3K almost totally prevented curcumin-induced HO-1 expression. Interestingly, NAC that also inhibited the increase of HO-1 levels, probably by preventing the generation of oxidative stress and redox cycling by curcumin, also decreased p-Akt expression levels when compared with related controls. It seems, therefore, there is some crosstalk between redox state and PI3K/Akt pathway, which may further help to modulate Nrf2 signaling by curcumin. In fact, it is known that ROS may impact on MAPK/ERK and PI3K/Akt pathways by facilitating activation of kinase cascades and/or by inhibiting the activity of phosphatases such as PTEN [60, 61]. It was shown that the protein Kras is directly activated by $S$-glutathionylation of a reactive thiol group due to the increased levels of oxidants, which triggers downstream signaling through phosphorylation of ERK and Akt [62, 63]. This may have happened with curcumin treatment as well as the inhibition of the phosphatase PTEN (a negative regulator of Akt activity), inducing Akt activity. In fact, it was also previously shown that the capacity of curcumin to induce HO-1 was accompanied by a decrease of phosphatase activities [17].

The induction of p-Akt levels was, however, largely dependent on serum stimulation during curcumin incubation. Mitogenic signals, present in the serum, stimulate the activation of receptor tyrosine kinases that results in a downstream activation of class I PI3K [57, 60]. PI3K activation results in phosphorylation (activation) of Akt, a reaction that can be reversed by the PIP3 phosphatase PTEN, which than modulates its cellular targets involved in cell survival, growth, proliferation and metabolism [57]. With curcumin incubation, p-Akt levels in serum-stimulated cells were increased and persisted with time. Curcumin incubation without serum stimulation did not change significantly p-Akt levels but induced the expression of HO-1, albeit to a smaller extent. This indicates that the PI3K is important in the upregulation of HO- 1 expression by curcumin, but is not totally dependent on it. Therefore, the induction of a stress response by curcumin may be elevated in cells under mitogenic signaling, and in cells with potential to proliferate, such as fibroblasts. For example, during wound healing proliferation of skin fibroblasts is induced, and cells are subject to stress elicited by inflammation triggered by toxic-free heme released by hemolysis, resulting in the induction of HO-1 in the injured tissue [64]. Therefore, an increased stress response in this situation elicited by curcumin may be beneficial by increasing the expression of HO-1, important in the detoxification of heme groups and in the process of wound healing [64]. In fact, it has been previously shown that curcumin enhanced the process of wound healing in cultured cells and in rats $[9,65]$.

In conclusion, in this report we have shown that curcumin induces cellular stress responses in normal human skin fibroblasts through impairment of cellular thiol-disulfide redox state. This curcumin-induced GSH redox stress leads to a strong induction of HO-1 and other antioxidant enzymes as well as an increase in cellular GSH levels, most probably through Nrf2 signaling. The hormetic induction of a stress response elicited by the hormetin curcumin led to increased protection against a further oxidant challenge, 
supporting the view that mild stress-induced hormesis can be applied for the modulation of aging and for improving the cellular functionality.

This work was supported by the Foundation for Science and Technology, Portugal, through the research grant PTDC/QUIBIQ/101392/2008 (NaturAge), which is co-funded by the program COMPETE from QREN with co-participation from the European Community fund FEDER. The authors thank Barbara Dymek for her valuable help in the MTT assay experiments.

The authors have declared no conflict of interest.

\section{References}

[1] Stanner, S. A., Hughes, J., Kelly, C. N., Buttriss, J., A review of the epidemiological evidence for the "antioxidant hypothesis". Public Health Nutr. 2004, 7, 407-422.

[2] Mattson, M. P., Hormesis defined. Ageing Res. Rev. 2008, 7, 1-7.

[3] Rattan, S. I. S., Hormesis in aging. Ageing Res. Rev. 2008, 7, 63-78.

[4] Goel, A., Kunnumakkara, A. B., Aggarwal, B. B., Curcumin as "Curecumin": from kitchen to clinic. Biochem. Pharmacol. 2008, 75, 787-809.

[5] Rattan, S. I. S., Increased molecular damage and heterogeneity as the basis of aging. Biol. Chem. 2008, 389, 267-272.

[6] Rattan, S. I. S., Clark, B. F., Understanding and modulating ageing. IUBMB Life 2005, 57, 297-304.

[7] Calabrese, E. J., Bachmann, K. A., Bailer, A. J., Bolger, P. M. et al., Biological stress response terminology: integrating the concepts of adaptive response and preconditioning stress within a hormetic dose-response framework. Toxicol. Appl. Pharmacol. 2007, 222, 122-128.

[8] Rattan, S. I. S., in: Le Bourg, E., Rattan, S. I. S. (Eds.), Mild Stress and Healthy Aging: Applying Hormesis in Aging Research and Interventions, Springer Science+Business Media B. V., Berlin 2008, pp. 81-96.

[9] Rattan, S. I. S., Fernandes, R. A., Demirovic, D., Dymek, B., Lima, C. F., Heat stress and hormetin-induced hormesis in human cells: effects on aging, wound healing, angiogenesis, and differentiation. Dose Response 2009, 7, 90-103.

[10] Kato, K., Ito, H., Kamei, K., Iwamoto, I., Stimulation of the stress-induced expression of stress proteins by curcumin in cultured cells and in rat tissues in vivo. Cell Stress Chaperones 1998, 3, 152-160.

[11] Teiten, M. H., Reuter, S., Schmucker, S., Dicato, M., Diederich, M., Induction of heat shock response by curcumin in human leukemia cells. Cancer Lett. 2009, 279, 145-154.

[12] Ali, R. E., Rattan, S. I., Curcumin's biphasic hormetic response on proteasome activity and heat-shock protein synthesis in human keratinocytes. Ann. NY Acad. Sci. 2006, 1067, 394-399.

[13] Scapagnini, G., Colombrita, C., Amadio, M., D'Agata, V. et al., Curcumin activates defensive genes and protects neurons against oxidative stress. Antioxid. Redox Signal. 2006, 8, 395-403.

[14] Motterlini, R., Foresti, R., Bassi, R., Green, C. J., Curcumin, an antioxidant and anti-inflammatory agent, induces heme oxygenase- 1 and protects endothelial cells against oxidative stress. Free Radic. Biol. Med. 2000, 28, 1303-1312.

[15] Andreadi, C. K., Howells, L. M., Atherfold, P. A., Manson, M. M., Involvement of Nrf2, p38, B-Raf, and nuclear factorkappaB, but not phosphatidylinositol 3-kinase, in induction of hemeoxygenase- 1 by dietary polyphenols. Mol. Pharmacol. 2006, 69, 1033-1040.

[16] Kang, E. S., Woo, I. S., Kim, H. J., Eun, S. Y. et al., Upregulation of aldose reductase expression mediated by phosphatidylinositol 3-kinase/Akt and Nrf2 is involved in the protective effect of curcumin against oxidative damage. Free Radic. Biol. Med. 2007, 43, 535-545.

[17] McNally, S. J., Harrison, E. M., Ross, J. A., Garden, O. J., Wigmore, S. J., Curcumin induces heme oxygenase 1 through generation of reactive oxygen species, p38 activation and phosphatase inhibition. Int. J. Mol. Med. 2007, 19, 165-172.

[18] Lavoie, S., Chen, Y., Dalton, T. P., Gysin, R. et al., Curcumin, quercetin, and $\mathrm{tBHO}$ modulate glutathione levels in astrocytes and neurons: importance of the glutamate cysteine ligase modifier subunit. J. Neurochem. 2009, 108, 1410-1422.

[19] Ye, S. F., Hou, Z. Q., Zhong, L. M., Zhang, Q. Q., Effect of curcumin on the induction of glutathione S-transferases and $\mathrm{NADP}(\mathrm{H})$ :quinone oxidoreductase and its possible mechanism of action. Yao Xue Xue Bao 2007, 42, 376-380.

[20] Balogun, E., Hoque, M., Gong, P., Killeen, E. et al., Curcumin activates the haem oxygenase-1 gene via regulation of $\mathrm{Nrf} 2$ and the antioxidant-responsive element. Biochem. J. 2003, 371, 887-895.

[21] Shen, G., Xu, C., Hu, R., Jain, M. R. et al., Modulation of nuclear factor E2-related factor 2-mediated gene expression in mice liver and small intestine by cancer chemopreventive agent curcumin. Mol. Cancer Ther. 2006, 5, 39-51.

[22] Garg, R., Gupta, S., Maru, G. B., Dietary curcumin modulates transcriptional regulators of phase I and phase II enzymes in benzo[a]pyrene-treated mice: mechanism of its anti-initiating action. Carcinogenesis 2008, 29, 1022-1032.

[23] Farombi, E. O., Shrotriya, S., Na, H. K., Kim, S. H., Surh, Y. J., Curcumin attenuates dimethylnitrosamine-induced liver injury in rats through Nrf2-mediated induction of heme oxygenase-1. Food Chem. Toxicol. 2008, 46, 1279-1287.

[24] Nguyen, T., Nioi, P., Pickett, C. B., The Nrf2-antioxidant response element signaling pathway and its activation by oxidative stress. J. Biol. Chem. 2009, 284, 13291-13295.

[25] Surh, Y. J., Kundu, J. K., Na, H. K., Nrf2 as a master redox switch in turning on the cellular signaling involved in the induction of cytoprotective genes by some chemopreventive phytochemicals. Planta Medica 2008, 74, 1526-1539. 
[26] Kraft, D. C., Deocaris, C. C., Rattan, S. I. S., Proteasomal oscillation during mild heat shock in aging human skin fibroblasts. Ann. NY Acad. Sci. 2006, 1067, 224-227.

[27] Lima, C. F., Andrade, P. B., Seabra, R. M., FernandesFerreira, M., Pereira-Wilson, C., The drinking of a Salvia officinalis infusion improves liver antioxidant status in mice and rats. J. Ethnopharmacol. 2005, 97, 383-389.

[28] Lima, C. F., Fernandes-Ferreira, M., Pereira-Wilson, C., Phenolic compounds protect HepG2 cells from oxidative damage: relevance of glutathione levels. Life Sci. 2006, 79, 2056-2068.

[29] Lima, C. F., Carvalho, F., Fernandes, E., Bastos, M. L. et al., Evaluation of toxic/protective effects of the essential oil of Salvia officinalis on freshly isolated rat hepatocytes. Toxicol. In Vitro 2004, 18, 457-465.

[30] McMahon, M., Itoh, K., Yamamoto, M., Chanas, S. A. et al., The Cap' $n$ 'Collar basic leucine zipper transcription factor Nrf2 (NF-E2 p45-related factor 2) controls both constitutive and inducible expression of intestinal detoxification and glutathione biosynthetic enzymes. Cancer Res. 2001, 61, 3299-3307.

[31] Li, W., Kong, A. N., Molecular mechanisms of Nrf2-mediated antioxidant response. Mol. Carcinog. 2009, 48, 91-104.

[32] Ferrari, C. K., Functional foods, herbs and nutraceuticals: towards biochemical mechanisms of healthy aging. Biogerontology 2004, 5, 275-289.

[33] Barone, E., Calabrese, V., Mancuso, C., Ferulic acid and its therapeutic potential as a hormetin for age-related diseases. Biogerontology 2009, 10, 97-108.

[34] Le Bourg, E., Rattan, S. I. S., (Eds.), Mild Stress and Healthy Aging: Applying Hormesis in Aging Research and Interventions, Springer Science+Business Media B. V., Berlin 2008.

[35] Ballatori, N., Krance, S. M., Notenboom, S., Shi, S. et al., Glutathione dysregulation and the etiology and progression of human diseases. Biol. Chem. 2009, 390, 191-214.

[36] Kang, M. J., Kim, H. J., Kim, H. K., Lee, J. Y. et al., The effect of age and calorie restriction on HIF-1-responsive genes in aged liver. Biogerontology 2005, 6, 27-37.

[37] Patriarca, S., Furfaro, A. L., Cosso, L., Pesce Maineri, E. et al., Heme oxygenase 1 expression in rat liver during ageing and ethanol intoxication. Biogerontology 2007, 8, 365-372.

[38] Bloomer, S. A., Zhang, H. J., Brown, K. E., Kregel, K. C., Differential regulation of hepatic heme oxygenase-1 protein with aging and heat stress. J. Gerontol. A Biol. Sci. Med. Sci. 2009, 64, 419-425.

[39] Ito, Y., Betsuyaku, T., Moriyama, C., Nasuhara, Y., Nishimura, M., Aging affects lipopolysaccharide-induced upregulation of heme oxygenase- 1 in the lungs and alveolar macrophages. Biogerontology 2009, 10, 173-180.

[40] Mulcahy, R. T., Wartman, M. A., Bailey, H. H., Gipp, J. J., Constitutive and beta-naphthoflavone-induced expression of the human gamma-glutamylcysteine synthetase heavy subunit gene is regulated by a distal antioxidant response element/TRE sequence. J. Biol. Chem. 1997, 272, 7445-7454.
[41] Dinkova-Kostova, A. T., Talalay, P., Direct and indirect antioxidant properties of inducers of cytoprotective proteins. Mol. Nutr. Food Res. 2008, 52, S128-S138.

[42] Dinkova-Kostova, A. T., Massiah, M. A., Bozak, R. E., Hicks, R. J., Talalay, P., Potency of Michael reaction acceptors as inducers of enzymes that protect against carcinogenesis depends on their reactivity with sulfhydryl groups. Proc. Natl. Acad. Sci. USA 2001, 98, 3404-3409.

[43] Suh, J. H., Shenvi, S. V., Dixon, B. M., Liu, H. et al., Decline in transcriptional activity of Nrf2 causes age-related loss of glutathione synthesis, which is reversible with lipoic acid. Proc. Natl. Acad. Sci. USA 2004, 101, 3381-3386.

[44] Duan, W., Zhang, R., Guo, Y., Jiang, Y. et al., Nrf2 activity is lost in the spinal cord and its astrocytes of aged mice. In Vitro Cell. Dev. Biol. Anim. 2009, 45, 388-397.

[45] Shih, P. H., Yen, G. C., Differential expressions of antioxidant status in aging rats: the role of transcriptional factor Nrf2 and MAPK signaling pathway. Biogerontology 2007, 8, 71-80.

[46] Keogh, B. P., Allen, R. G., Pignolo, R., Horton, J. et al., Expression of hydrogen peroxide and glutathione metabolizing enzymes in human skin fibroblasts derived from donors of different ages. J. Cell. Physiol. 1996, 167, 512-522.

[47] Rebrin, I., Kamzalov, S., Sohal, R. S., Effects of age and caloric restriction on glutathione redox state in mice. Free Radic. Biol. Med. 2003, 35, 626-635.

[48] Das, S., Mandal, A. K., Ghosh, A., Panda, S. et al., Nanoparticulated quercetin in combating age related cerebral oxidative injury. Curr. Aging Sci. 2008, 1, 169-174.

[49] Aydin, S., Atukeren, P., Cakatay, U., Uzun, H., Altug, T., Gender-dependent oxidative variations in liver of aged rats. Biogerontology 2010, 11, 335-346.

[50] Kitani, K., Osawa, T., Yokozawa, T., The effects of tetrahydrocurcumin and green tea polyphenol on the survival of male C57BL/6 mice. Biogerontology 2007, 8, 567-573.

[51] Bala, K., Tripathy, B. C., Sharma, D., Neuroprotective and anti-ageing effects of curcumin in aged rat brain regions. Biogerontology 2006, 7, 81-89.

[52] Toussaint, O., Medrano, E. E., von Zglinicki, T., Cellular and molecular mechanisms of stress-induced premature senescence (SIPS) of human diploid fibroblasts and melanocytes. Exp. Gerontol. 2000, 35, 927-945.

[53] Nogueira, V., Park, Y., Chen, C. C., Xu, P. Z. et al., Akt determines replicative senescence and oxidative or oncogenic premature senescence and sensitizes cells to oxidative apoptosis. Cancer Cell. 2008, 14, 458-470.

[54] Sykiotis, G. P., Bohmann, D., Keap1/Nrf2 signaling regulates oxidative stress tolerance and lifespan in Drosophila. Dev. Cell 2008, 14, 76-85.

[55] Colombrita, C., Calabrese, V., Stella, A. M., Mattei, F. et al., Regional rat brain distribution of heme oxygenase-1 and manganese superoxide dismutase mRNA: relevance of redox homeostasis in the aging processes. Exp. Biol. Med. (Maywood) 2003, 228, 517-524.

[56] Hirose, W., Ikematsu, K., Tsuda, R., Age-associated increases in heme oxygenase-1 and ferritin immunoreactivity 
in the autopsied brain. Leg. Med. (Tokyo) 2003, 5, S360-S366.

[57] Manning, B. D., Cantley, L. C., AKT/PKB signaling: navigating downstream. Cell 2007, 129, 1261-1274.

[58] Jiang, B. H., Liu, L. Z., PI3K/PTEN signaling in angiogenesis and tumorigenesis. Adv. Cancer Res. 2009, 102, 19-65.

[59] Pugazhenthi, S., Akhov, L., Selvaraj, G., Wang, M., Alam, J., Regulation of heme oxygenase- 1 expression by demethoxy curcuminoids through Nrf2 by a PI3-kinase/Akt-mediated pathway in mouse beta-cells. Am. J. Physiol. Endocrinol. Metab. 2007, 293, E645-E655.

[60] Burhans, W. C., Heintz, N. H., The cell cycle is a redox cycle: linking phase-specific targets to cell fate. Free Radic. Biol. Med. 2009, 47, 1282-1293.

[61] Janssen-Heininger, Y. M., Mossman, B. T., Heintz, N. H., Forman, H. J. et al., Redox-based regulation of signal transduction: principles, pitfalls, and promises. Free Radic. Biol. Med. 2008, 45, 1-17.

[62] Dalle-Donne, I., Rossi, R., Giustarini, D., Colombo, R., Milzani, A., S-glutathionylation in protein redox regulation. Free Radic. Biol. Med. 2007, 43, 883-898.

[63] Clavreul, N., Adachi, T., Pimental, D. R., Ido, Y. et al., S-glutathiolation by peroxynitrite of p21ras at cysteine-118 mediates its direct activation and downstream signaling in endothelial cells. FASEB J. 2006, 20, 518-520.

[64] Grochot-Przeczek, A., Lach, R., Mis, J., Skrzypek, K. et al., Heme oxygenase- 1 accelerates cutaneous wound healing in mice. PLoS One 2009, 4, e5803.

[65] Panchatcharam, M., Miriyala, S., Gayathri, V. S., Suguna, L., Curcumin improves wound healing by modulating collagen and decreasing reactive oxygen species. Mol. Cell. Biochem. 2006, 290, 87-96. 